\title{
Preparation of organic-ceramic-metal multihybrid particles and their organized assembly
}

\author{
Jun Matsui ${ }^{1,2}$, Salina Parvin ${ }^{1}$, Eriko Sato $^{1,3}$ and Tokuji Miyashita ${ }^{1}$
}

This paper describes the synthesis and assembly of multihybrid particles having a gold core, silica as an initial shell and polymethylmethacrylate (pMMA) as a second shell (Au@SiO $\left.{ }_{2} @ \mathrm{pMMA}\right)$. Multihybrid particles were synthesized in a stepwise

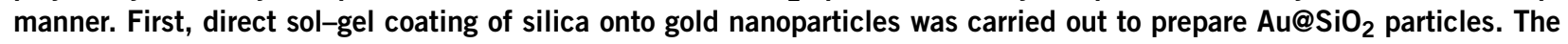
$\mathrm{Au}_{\mathrm{SiO}}$ particles were modified with 3-(triethoxysilyl)propyl 2-bromo-2-methylpropanoate (BMPS), which is an initiator for atom transfer radical polymerization (ATRP) of MMA. Finally, bulk ATRP of MMA was conducted using BMPS-coated Au@SiO 2 particles. Au@SiO $\mathrm{S}_{2} @$ pMMA particles formed a closely packed monolayer at the air-water interface. The monolayer can be deposited onto a solid substrate using the Langmuir-Blodgett technique. Atomic force microscopy and scanning electron microscopy images of the deposited film show a uniform and closely packed monolayer film in an area a few tens-of-micrometers in size with no voids. Moreover, a clear Au core, silica shell and pMMA shell structure were observed in a transmission electron microscopy image of the transferred film. It is particularly interesting that the monolayer film shows no clear plasmon absorption of the gold core, as observed in the chloroform solution of the particle. Results show that a thick silica shell masked the monolayer film's plasmon absorption.

Polymer Journal (2010) 42, 142-147; doi:10.1038/pj.2009.320; published online 23 December 2009

Keywords: hybrid materials; langmuir-blodgett film; particle

\section{INTRODUCTION}

Organic-inorganic hybrid nanomaterials seem to be a creative alternative for obtaining new materials with unusual features that cannot be achieved by any single component. Nanometer-scale combinations of organic and inorganic materials can yield, for example, flexible films with high mechanical and thermal stability or flexible films with magnetic or electrical properties. ${ }^{1,2}$ In contrast, metal nanoparticles have attracted much interest because of their unique optical properties based on localized surface plasmon resonance, which has wide applicability for optical filters, ${ }^{3}$ immunoassay labeling ${ }^{4}$ and Raman spectroscopy enhancers. ${ }^{5}$ Gold nanoparticles are probably the most studied and established system because of tremendous advances in their synthesis procedures. ${ }^{6}$ The optical properties of gold nanoparticles can be further controlled by coating the surface with a dielectric material and by changing the interparticle spacing. ${ }^{7}$ For that reason, synthesis of hybrid gold particles that have organic or inorganic shells and assembly of hybrid gold particles into two-dimensional and three-dimensional orders have become active areas of research. For example, Heath et al. ${ }^{8}$ studied the optical properties of a hybrid goldnanoparticle monolayer at the air-water interface by changing the interparticle distance with surface pressure. Fukuda and others ${ }^{9}$ controlled the interparticle distance of gold nanoparticles by changing the polymer shell thickness. Xia and others ${ }^{10}$ synthesized a gold nanoparticle with a silica shell and assembled the hybrid particles in an array to produce a plasmonic waveguide. These reported studies are related to the synthesis and assembly of hybrid particles composed of a gold core with a single shell. Few reports describe hybrid particles composed of a single gold core with a multiple-layer shell. ${ }^{11}$ The assembly of such gold particles has not been reported.

For this study, we prepared multihybrid spherical particles, which have an Au core, silica as an initial shell and polymethylmethacrylate (pMMA) as a second shell (Au@SiO $@$ @MMA), and prepared two-dimensional and three-dimensional assemblies of the particle using the Langmuir-Blodgett (LB) technique. Silica-coated gold particles $\left(\mathrm{Au} @ \mathrm{SiO}_{2}\right)$ were prepared by direct coating of gold nanoparticles through a sol-gel reaction of tetraethylorthosilicate (TEOS). Thereafter, Au@SiO 2 was coated with an initiator for atom transfer radical polymerization (ATRP). The ATRP of MMA was initiated from the initiator-coated particle. The resulting Au@SiO ${ }_{2} @$ pMMA particles were characterized using dynamic light scattering (DLS) and transmission electron microscopy (TEM) measurements. Then, an $\mathrm{Au} @ \mathrm{SiO}_{2} @$ pMMA particle film was prepared at the air-water interface, and the particle film was transferred onto a solid substrate. The morphology of the film was observed using atomic force microscopy

${ }^{1}$ Institute of Multidisciplinary Research for Advanced Materials, Tohoku University, Aoba-ku, Sendai, Japan and ${ }^{2}$ Precursory Research for Embryonic Science and Technology, Japan Science and Technology Agency, Honcho, Kawaguchi, Japan

${ }^{3}$ Current address: Department of Applied Chemistry and Bioengineering, Graduate School of Engineering, Osaka City University, Sugimoto, Sumiyoshi-ku, Osaka 558-8585, Japan.

Correspondence: Professor T Miyashita, Institute of Multidisciplinary Research for Advanced Materials, Tohoku University, 2-1-1 Katahira Aoba-ku, Sendai, Miyagi 980-8577, Japan. E-mail: miya@tagen.tohoku.ac.jp

Received 13 August 2009; revised 6 October 2009; accepted 22 October 2009; published online 23 December 2009 
(AFM), scanning electron microscopy (SEM) and TEM. In addition, optical properties of the multilayer film were elucidated using ultraviolet-visible (UV-Vis) absorption measurements.

\section{EXPERIMENTAL PROCEDURE}

Materials

For use in this study, $\mathrm{HAuCl}_{4} 3 \mathrm{H}_{2} \mathrm{O}$ (98\%), 4,4-dinonyl-2-2,2-bipyridine (dNbipy, 97\%) and $\mathrm{Cu}(\mathrm{I}) \mathrm{Cl}$ (99.99\%) were purchased from Aldrich (St Louis, MO, USA). Ethyl-2-bromoisobutyrate was purchased from TCI (Tokyo, Japan). Ammonia solution (28\%) and trisodium citrate dihydrate (Na-cit, 99\%) were purchased from Wako Pure Chemical Industries (Osaka, Japan). TEOS and octadecyltrichlorosilane were purchased from Shinetsu Chemical (Tokyo, Japan). All chemicals were used as received. In addition, 3-(triethoxysilyl)propyl 2-bromo-2-methylpropanoate (BMPS) was kindly donated by Chisso (Tokyo, Japan). MMA was obtained from Nacalai Tesque (Kyoto, Japan) and purified by distillation under reduced pressure. Ultrapure deionized water was prepared (CPW-101; Advantec Toyo Kaisha, Tokyo, Japan). All other chemicals were purchased from TCI. All solvents were distilled before use.

\section{Synthesis of organic-inorganic-metal multihybrid nanoparticle (Au@SiO ${ }_{2} @$ pMMA)}

Organic-inorganic-metal multihybrid particles were synthesized using the stepwise procedure presented in Scheme 1. Each procedure is described below.

Synthesis of gold nanoparticles and silica coating on gold nanoparticles (step 1). Gold nanoparticles were prepared by a reduction of gold salt with Na-cit, following the reported procedure. ${ }^{12}$ Briefly, $1 \mathrm{ml}$ of freshly prepared $0.34 \mathrm{M} \mathrm{Na}$-cit aqueous solution was added to $200 \mathrm{ml}$ of $0.24 \mathrm{~mm} \mathrm{HAuCl}_{4}$ aqueous solution at boiling temperature with vigorous stirring. The mixture turned wine red within a few minutes, indicating the presence of gold nanoparticles. After $30 \mathrm{~min}$ of vigorous stirring, $5 \mathrm{ml}$ of $1 \mathrm{~mm} \mathrm{Na}$-cit was added again to the boiling gold sol. The mixture was stirred for another $1 \mathrm{~h}$. The prepared gold solution was cooled to room temperature. The sol-gel seeded-growth process was applied to produce a silica shell on the gold nanoparticle surface. ${ }^{13}$ Then, TEOS in ethanol $(5 \mathrm{~mm})$ was slowly added to the gold sol. The mixture was stirred for $10 \mathrm{~min}$, and $5 \mathrm{ml}$ of ammonia solution was added to the TEOS/ethanol/gold sol mixture with vigorous stirring. The reaction was stirred for $3 \mathrm{~h}$ to produce $\mathrm{Au} @ \mathrm{SiO}_{2}$ particles.

Coating of BMPS onto Au@SiO $\mathrm{S}_{2}$ particles and graft polymerization of MMA onto $\mathrm{Au} @ \mathrm{SiO}_{2}$ particles (step 2). The $\mathrm{Au} @ \mathrm{SiO}_{2}$ particles were separated from the colloidal solution with centrifugation $(4000$ r.p.m. $\times 90 \mathrm{~min})$ and diluted with ethanol $(30 \mathrm{ml}, 7.7 \mathrm{wt} \%)$. A mixture of ammonia solution $(14 \mathrm{ml})$ and ethanol $(175 \mathrm{ml})$ was added dropwise to the particles suspended in ethanol under stirring. The mixture was then stirred for $2 \mathrm{~h}$ at $40{ }^{\circ} \mathrm{C}$. BMPS $(2 \mathrm{~g}, 4.8 \mathrm{mmol})$ was then dissolved in ethanol $(10 \mathrm{ml})$ and added dropwise into the system.
The reaction mixture was stirred continuously for another $18 \mathrm{~h}$ at $40^{\circ} \mathrm{C}$. The initiator-modified $\mathrm{Au} @ \mathrm{SiO}_{2}$ particles were then washed by repeating the centrifugation-redispersion process using ethanol $(4000$ r.p.m. $\times 60 \mathrm{~min})$ to remove the free initiator from the surface of the particles. The procedure was repeated three times. Finally, the initiator-coated $\mathrm{Au} @ \mathrm{SiO}_{2}$ particles $\left(\mathrm{Au} @ \mathrm{SiO}_{2}\right.$ BMPS) were dried under vacuum at room temperature. Bulk polymerization of MMA was conducted from the $\mathrm{Au} @ \mathrm{SiO}_{2}$-BMPS particles. ${ }^{14}$ The $\mathrm{Au} @ \mathrm{SiO}_{2}-$ BMPS particles $(0.4 \mathrm{~g})$ were mixed with MMA $(9.8 \mathrm{~g}, 98 \mathrm{mmol})$ and purged with argon. Then, ethyl-2-bromoisobutyrate, which acts as a 'sacrificial' free initiator $(3.2 \mathrm{mg}, 0.016 \mathrm{mmol}), \mathrm{Cu}(\mathrm{I}) \mathrm{Cl}(16 \mathrm{mg}, 0.16 \mathrm{mmol})$ and dNbipy ( $134 \mathrm{mg}, 0.32 \mathrm{mmol}$ ) were added to the particles suspended in MMA under an argon atmosphere. The role of the free initiator is to accumulate an appropriate amount of $\mathrm{Cu}(\mathrm{II})$ species by the termination of propagating radicals and thus to control polymerization by the so-called persistent radical effect. ${ }^{15,16}$ Polymerization was conducted at $70{ }^{\circ} \mathrm{C}$ for $14 \mathrm{~h}$. The pMMA-grafted $\mathrm{Au} @ \mathrm{SiO}_{2}$ particles $\left(\mathrm{Au} @ \mathrm{SiO}_{2} @ \mathrm{pMMA}\right.$ ) were precipitated in 2 wt $\%$ citric acid/ methanol solution to remove the catalyst. The $\mathrm{Au} @ \mathrm{SiO}_{2} @ \mathrm{pMMA}$ particles were purified by repeating the centrifugation-redispersion process using tetrahydrofuran (4000 r.p.m. $\times 60 \mathrm{~min}$, five times) and drying them under vacuum at room temperature.

\section{Measurements}

The molecular weights of pMMA were determined using gel permeation chromatography (Tosoh, Tokyo, Japan) with a polystyrene standard. Fourier transform infrared (FT-IR) spectra were obtained with an FT-IR spectrometer (FT/IR-230; Jasco, Tokyo, Japan). UV-Vis spectra were obtained using a UV-visible absorption spectrometer (U-3000; Hitachi, Tokyo, Japan). Thermogravimetric-differential thermal analysis was conducted (Thermo plus TG 8120; Rigaku, Showajima, Japan). The size distributions of the particles were obtained with electrophoretic light scattering using the DLS method (ELS-8000; Otsuka Electronics, Hirakata, Japan). Microscopic images were taken and surface morphologies of the particles were assessed using TEM (JEM-3010; JEOL, Tachimachi, Japan), AFM (SPI400; Seiko Instruments, Chiba, Japan) and scanning electron microscopy (SEM) (JSM-7000F; JEOL). TEM images were obtained at an accelerating voltage of $300 \mathrm{kV}$. Particle suspensions were dropped onto a collodion-coated copper grid; TEM images of particles were obtained after evaporating the solvent. For measuring TEM images of particles in the LB film, the monolayer was transferred directly to the TEM grid, which was mounted on a silicon wafer. Samples for SEM and AFM measurements were prepared by transferring the monolayer onto a silicon wafer using the LB technique. Thereafter, SEM measurements were conducted at an accelerating voltage of $10 \mathrm{kV}$. Surface pressure-area $(\pi-\mathrm{A})$ isotherm measurements were determined and deposition of monolayers was conducted using a computer-controlled Langmuir trough system (FSD-110; USI Systems, Fukushima, Japan) at a compression speed of $14 \mathrm{~cm}^{2} / \mathrm{min}$ at $20^{\circ} \mathrm{C}$. The monolayers were transferred onto a substrate using vertical
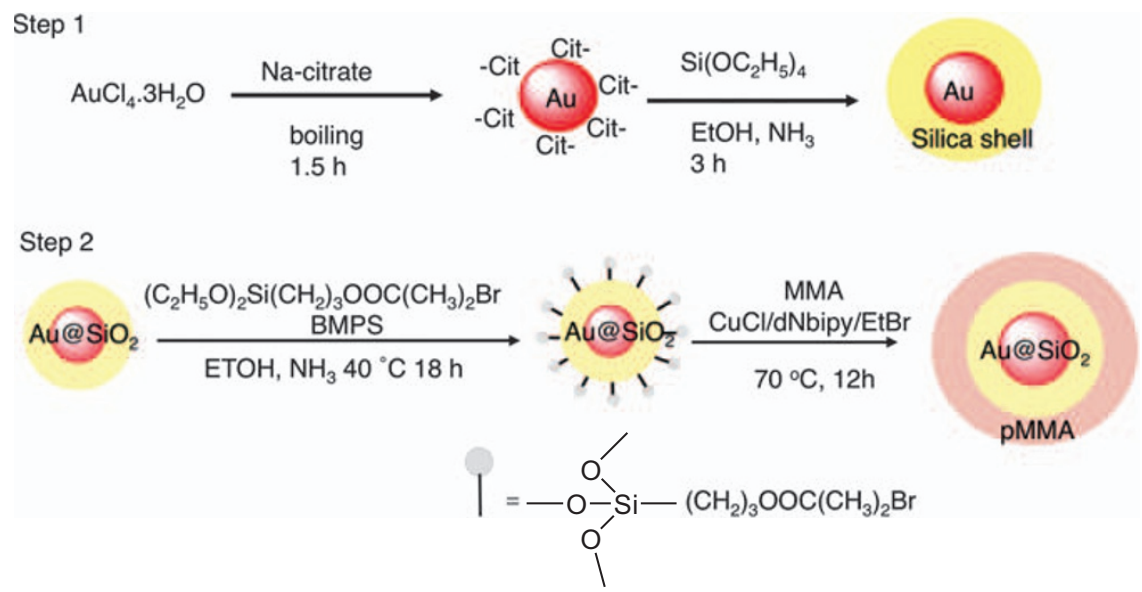

Scheme 1 Synthesis procedure for Au@SiO ${ }_{2} @$ pMMA. 
up-down deposition. Silicon wafers and quartz were used as deposition substrates. The substrates were first washed with 2-propanol; they were then treated with UV-O $\mathrm{O}_{3}$ cleaner (NL-UV 253; Filgen, Nagoya, Japan) to expose hydroxyl groups on the substrate surface. The cleaned hydrophilic substrates were immersed into an approximately $1 \times 10^{-6} \mathrm{M}$ octadecyltrichlorosilane chloroform solution to produce a hydrophobic substrate.

\section{RESULTS AND DISCUSSION}

Preparation of silica-coated gold nanoparticles $\left({\mathrm{Au} @ S i \mathrm{O}_{2}}_{2}\right)$

$\mathrm{Au} @ \mathrm{SiO}_{2}$ particles were prepared following the published method, ${ }^{13}$ with slight modifications. First, gold nanoparticles were prepared using sodium citrate as a reductant. ${ }^{12}$ To carry out silica coating on the surface of gold nanoparticles, a high concentration of citric acid was necessary. ${ }^{17-19}$ Therefore, the prepared citrate ion-coated gold nanoparticles were treated once again with $1 \mathrm{~mm}$ sodium citrate solution. It is known that during the preparation of gold nanoparticles, some of the citrate ions decompose to form acetone, dicarboxylic acid, itaconic acid and so on, which are adsorbed onto the surfaces of gold nanoparticles. ${ }^{17-19}$ After subsequent treatment of freshly prepared gold nanoparticles with additional citrate ions, fresh citrate ions can replace decomposed citrate ion products effectively, which results in a high concentration of citrate ion on the gold surface. Silica coating was first initiated by adsorption of silanol groups onto the nanoparticle's surface, leading to formation of a silica layer. The silica shell was grown with a sol-gel reaction of TEOS using ammonia as a catalyst. The average particle size estimated from DLS measurements was $130 \pm 40 \mathrm{~nm}$. The UV-Vis absorption spectrum of $\mathrm{Au} @ \mathrm{SiO}_{2}$ shows a broad peak centered at $520 \mathrm{~nm}$, which is attributed to the surface plasmon resonance band of gold nanoparticles (Figure 1). The large background absorption arises because of the scattering of light from the thick silica shell. The TEM image of $\mathrm{Au} @ S i O_{2}$ particles shows a clear core-shell structure (Figure 2). From the image, the darker and lighter parts of the particles were determined to be gold particles and the silica shell, respectively, because of differences in their electron densities.

\section{Graft polymerization of MMA onto Au@SiO 2 nanoparticles through ATRP}

$\mathrm{Au} @ \mathrm{SiO}_{2}$ particles were reacted with an ATRP initiator, BMPS, through a silane coupling reaction with hydroxy groups on the silica surface. The presence of BMPS moiety was confirmed by the FT-IR spectrum presented in Figure 3. After reacting with the BMPS initiator, particles show a characteristic ester-stretching peak at $1735 \mathrm{~cm}^{-1}$, and an alkyl-stretching peak at $2840-3000 \mathrm{~cm}^{-1}$, which are assignable to the BMPS initiator (Figure 3a). The amount of

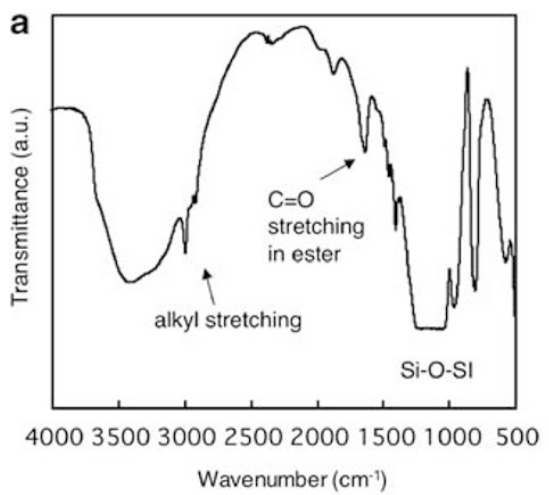

initiator incorporated onto the surface of particles was calculated as $3.76 \times 10^{-5} \mathrm{~mol} \mathrm{~m} \mathrm{~g}^{-1}$ by thermogravimetric-differential thermal analysis. The BMPS-coated $\mathrm{Au} @ \mathrm{SiO}_{2}$ particles were dispersed in MMA, and bulk ATRP of MMA was carried out. The FT-IR spectrum of particles after polymerization showed a large increase in the

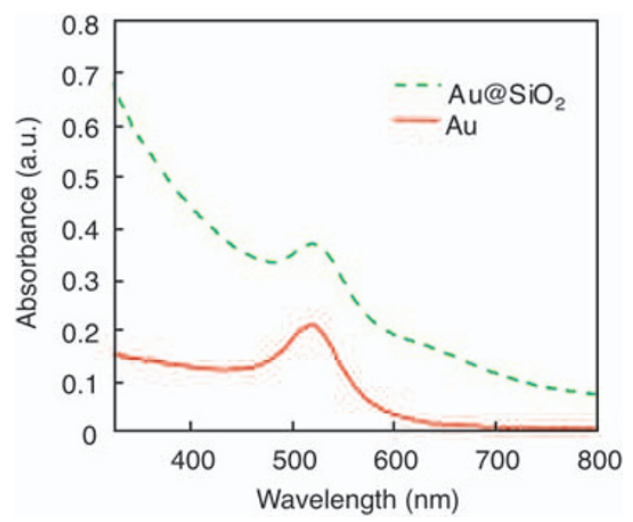

Figure 1 UV-Vis spectra of $\mathrm{Au}$ and $\mathrm{Au}_{\mathrm{S}} \mathrm{SiO}_{2}$ particles dispersed in an ethanol solution.

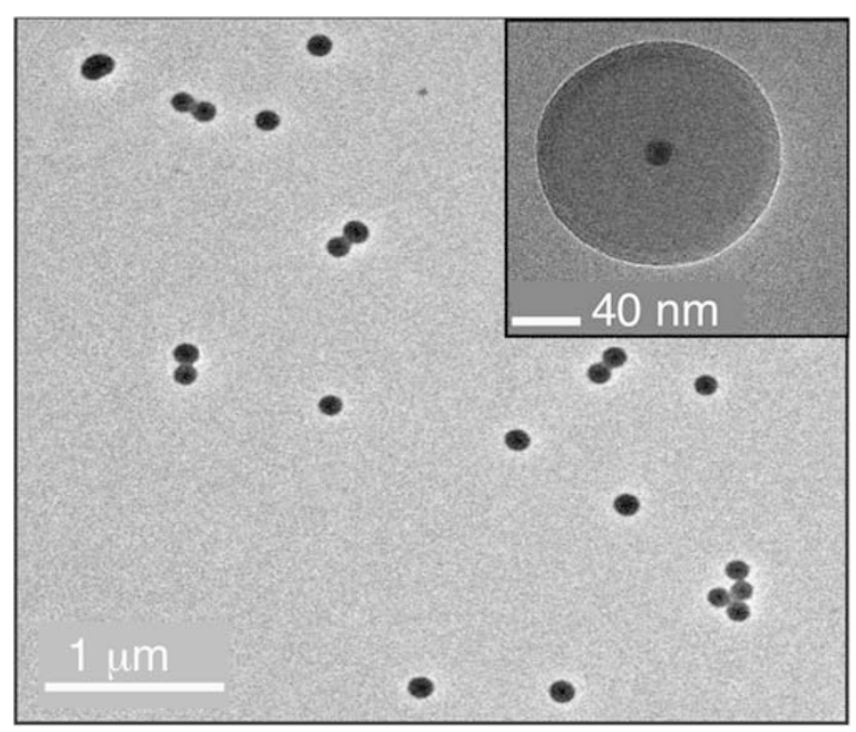

Figure 2 TEM image of $\mathrm{Au} @ \mathrm{SiO}_{2}$ particles dropped onto a collodion-coated TEM grid from an ethanol solution.

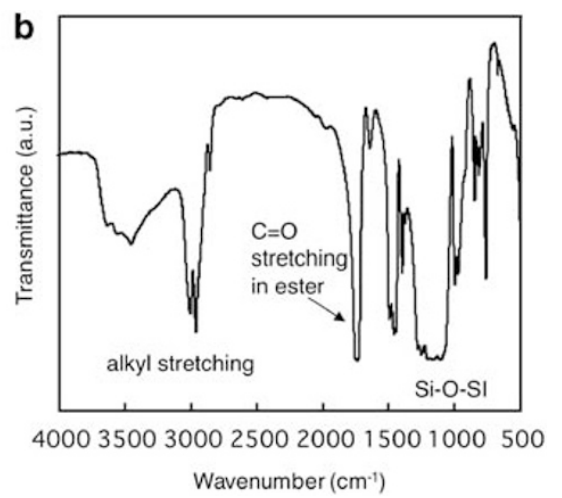

Figure 3 FT-IR spectra of (a) $\mathrm{Au} @ \mathrm{SiO}_{2}$-BMPS particles and (b) Au@SiO $@$ pMMA particles. 
intensity of the ester peak and of the alkyl peak compared with that of Si-O-Si $\left(1034-1249 \mathrm{~cm}^{-1}\right)$, which indicates the formation of a pMMA shell around the $\mathrm{Au} @ \mathrm{SiO}_{2}$ particles (Figure 3b). $\mathrm{Au} @ S_{1} \mathrm{O}_{2} @ \mathrm{pMMA}$ particles were well dispersed in good solvents for pMMA, such as chloroform and toluene, which supports the presence of pMMA in the second shell. The molecular weight of the grafted

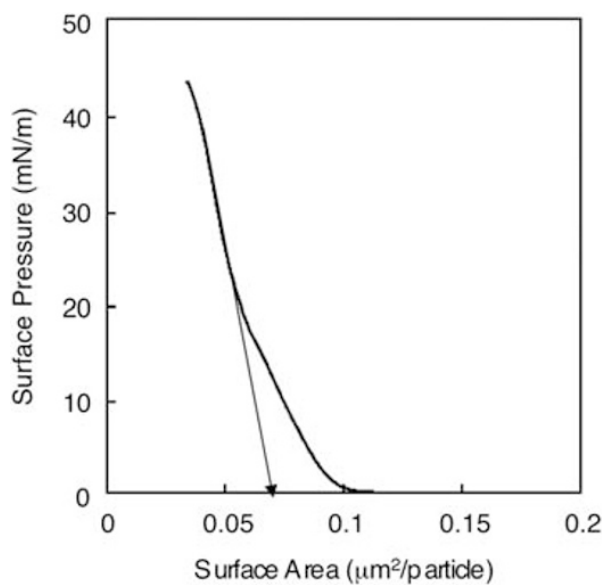

Figure $4 \pi$-A isotherm of Au@SiO ${ }_{2} @ p M M A$ particles measured at $20^{\circ} \mathrm{C}$. The straight arrow indicates the line extrapolated to determine the limiting surface area.
pMMA and the diameter of $\mathrm{Au} @ \mathrm{SiO}_{2} @ \mathrm{pMMA}$ were determined, respectively, using gel permeation chromatography and DLS. The molecular weight of the pMMA shell was determined to be $M n=47800$, with a polydispersity index of 1.45 , using a free polymer, which was produced simultaneously from the 'sacrificial' free initiator. Reportedly, in an ATRP system, the molecular weights of the free polymer and grafted polymers are nearly equal. ${ }^{20}$ The DLS measurements of $\mathrm{Au} @ \mathrm{SiO}_{2} @ \mathrm{pMMA}$ particles were determined in chloroform solution.The Au@SiO $\mathrm{S}_{2} @$ pMMA particle diameter was calculated as $284 \pm 80 \mathrm{~nm}$. The increase in the diameter to $150 \mathrm{~nm}$ indicates that an approximately 75-nm-thick second pMMA shell was coated onto the $\mathrm{Au} @ \mathrm{SiO}_{2}$ particle surface.

\section{Characterization of Au@SiO ${ }_{2} @$ pMMA nanoparticle LB film}

The Au@SiO ${ }_{2} @$ pMMA particles that dispersed in chloroform solution were spread onto a water surface, and the monolayer property of the particles was studied using $\pi$-A isotherm measurements (Figure 4). The isotherm shows a phase transition of around $0.06 \mu \mathrm{m}^{2}$ per particles and a high collapse pressure of $43 \mathrm{mN} \mathrm{m}^{-1}$. The limiting surface area per particle was determined by extrapolating the linear portion of the steep rise in the $\pi$-A isotherm to zero surface pressure. The estimated surface area of an $\mathrm{Au} @ \mathrm{SiO}_{2} @ \mathrm{pMMA}$ particle is $0.07 \mu \mathrm{m}^{2}$ per particle, which is close to the cross-sectional area calculated from the particle diameter, assuming that all particles are spherical with a $280 \mathrm{~nm}$ diameter $\left(0.06 \mu \mathrm{m}^{2}\right)$. These results indicate that Au@SiO $2 @$ pMMA particles form a closely packed monolayer at
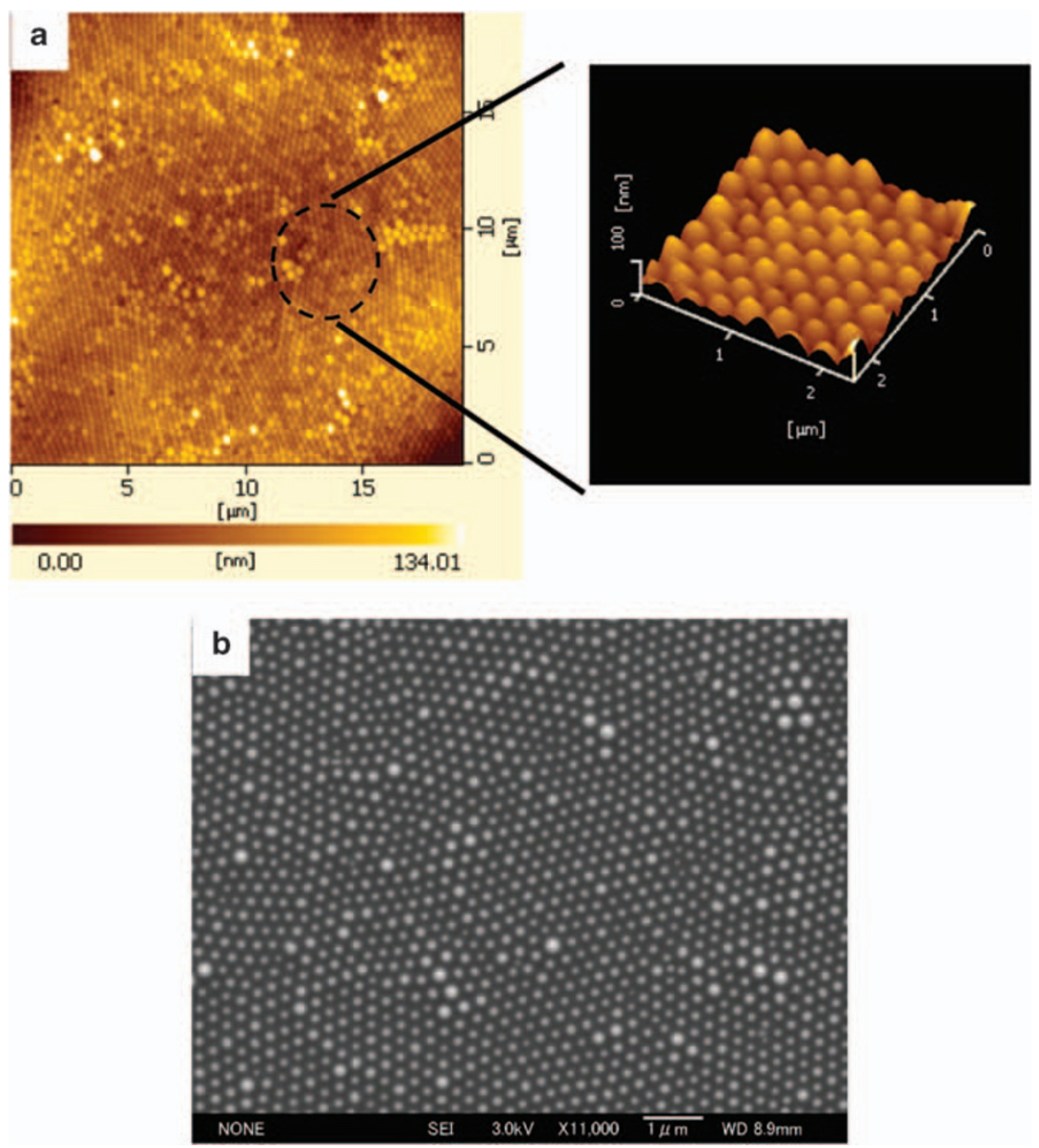

Figure 5 (a) AFM and (b) SEM images of a Au@SiO ${ }_{2} @ \mathrm{pMMA}$ particle monolayer deposited onto a silicon substrate at $30 \mathrm{mN} \mathrm{m}^{-1}$. 
the air-water interface. It is difficult to prepare a stable $\mathrm{Au} @ \mathrm{SiO}_{2}$ monolayer at the air-water interface because of the hydrophilicity of the silica shell.

One layer of the particle film was transferred onto a silicon wafer at $30 \mathrm{mN} \mathrm{m}^{-1}$; surface morphology was observed using AFM, SEM and TEM. The AFM and SEM images show a uniform and closely packed structure of several tens-of-micrometers in area with no voids (Figures $5 \mathrm{a}$ and $\mathrm{b}$ ). The high deposition pressure induced the multihybrid particle to form the closely packed structure in such a large area. The TEM image of the particle monolayer shows a clear Au core, a $\mathrm{SiO}_{2}$ first shell and a pMMA second shell structure because of differences in the electron density of gold, silica and pMMA (Figure 6). Polymer shell thickness is calculated from the distance between particles. The distance is estimated as $60-70 \mathrm{~nm}$, which indicates that the pMMA thickness is approximately $30 \mathrm{~nm}$. Conversely, the pMMA shell thickness at the edge of the assembly is about $60 \mathrm{~nm}$, which indicates that

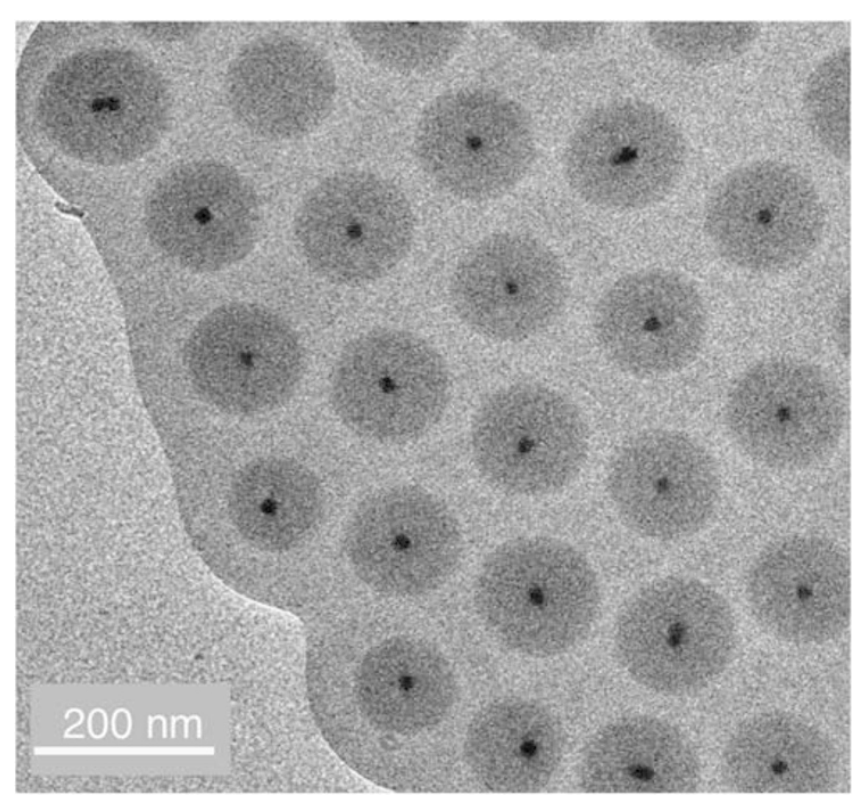

Figure 6 TEM images of one layer of Au@SiO2@pMMA particles deposited onto a collodion-coated TEM grid at $30 \mathrm{mN} \mathrm{m}^{-1}$.

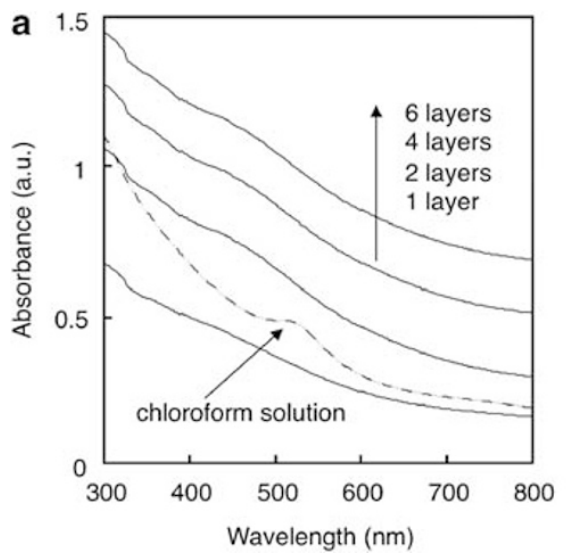

the pMMA shell takes a stretched conformation at the edge of the assembly. The thinner pMMA shell signifies that the pMMA shell between particles was compressed by surface pressure. During compression, some chains might interdigitate because of the attractive force occurring between chains. ${ }^{21}$ A few particles contained two gold nanoparticles in one shell. We concluded that the occurrence of this irregular particle is a few percent, because the absorption spectra of the multihybrid particle dispersed in chloroform show negligible absorption around $780 \mathrm{~nm}$, which is related to the plasmon coupling absorption of two gold nanoparticles.

A multilayer film of $\mathrm{Au} @ \mathrm{SiO}_{2} @ \mathrm{pMMA}$ was fabricated through a sequential deposition of the monolayer using the LB technique. The UV-Vis absorption spectra of a Au@SiO ${ }_{2} @$ pMMA monolayer deposited on a quartz substrate with different numbers of layers are portrayed in Figure 7a. It is particularly interesting that UV-Vis spectra do not show a clear surface plasmon absorption band, which is visible

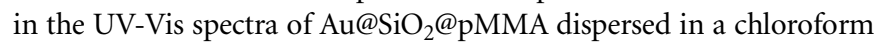
solution. The refractive index difference between air and silica is greater than that between chloroform and silica. Therefore, a high scattering of light occurs in the multilayer film. The high scattering of light masked the surface plasmon resonance absorption completely. ${ }^{22}$ Absorption does not linearly increase concomitantly with the increasing number of layers (Figure $7 \mathrm{~b}$ ). Transfer ratios of the multihybrid particle monolayer were 1.2 at the downstroke (first layer) and 1.1 at the upstroke (second layer) deposition. However, the upstroke deposition decreased to 0.7 in the fourth and sixth layer depositions (Table 1). The weak hydrophilic-hydrophilic interaction in the pMMA shell decreases the deposition amount concomitantly with an increase in the number of deposited layers. Therefore, polymers of

Table 1 Transfer ratios of $\mathrm{Au} @ \mathrm{SiO}_{2} @ \mathrm{pMMA}$ monolayer at different number of layers

\begin{tabular}{lcc}
\hline & \multicolumn{2}{c}{ Transfer ratio } \\
\cline { 2 - 3 } Layer number & Downstroke & Upstroke \\
\hline $1-2$ & 1.2 & 1.1 \\
$3-4$ & 1.2 & 0.7 \\
$5-6$ & 1.1 & 0.7 \\
\hline
\end{tabular}

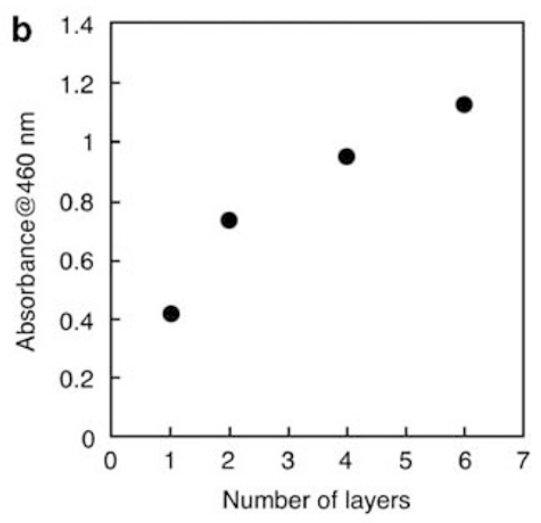

Figure 7 (a) UV-Vis spectra of Au@SiO ${ }_{2} @ p M M A$ particles dispersed in chloroform solution and Langmuir-Blodgett films with different numbers of layers. (b) Absorption intensity at $460 \mathrm{~nm}$ as a function of the number of layers. 
other types are necessary to achieve a uniform multilayer deposition. Possible candidates are polyalkylacrylamide and polyalkylmethacrylamide, which form a stable polymer monolayer because of hydrogen bonding in the amide groups. ${ }^{23,24}$ However, it is difficult to synthesize high-molecular-weight polymers by ATRP with alkylacrylamide and alkylmethacrylamide polymers. ${ }^{25}$ A possible procedure is the use of a reactive polymer as an initial shell, with subsequent conversion of the polymer to an alkylmethacrylamide polymer. $^{26,27}$

\section{CONCLUSION}

Multihybrid particles of $\mathrm{Au} @ \mathrm{SiO}_{2} @ \mathrm{pMMA}$ composed of a gold core, a silica shell and a pMMA second shell were synthesized through direct silica coating of gold nanoparticles, with subsequent ATRP of the MMA. The $\pi$-A isotherm of the multihybrid particles shows a steep rise in surface pressure with a high collapse pressure. The limiting surface area of the monolayer was close to the calculated crosssectional area of the particle, indicating that $\mathrm{Au} @ \mathrm{SiO}_{2} @ \mathrm{pMMA}$ particles form a closely packed monolayer. The monolayer is transferrable onto a solid substrate; the closely packed structure was confirmed by microscopic images of the deposited film. Clear surface plasmon absorption was not observed in the multilayer film because the thick silica shell increases the scattering of light. By contrast, when the outer refractive index is as close to that of the silica (approximately 1.44 at $589 \mathrm{~nm})$ as it is to that of the chloroform $(1.45$ at $589 \mathrm{~nm})$, the multihybrid particle shows a clear absorption from gold nanoparticles. Therefore, the particle film might be useful as a vapor sensor that can detect a vapor with a refractive index of approximately 1.45 according to the change in film color.

\section{ACKNOWLEDGEMENTS}

This work was supported by a Grant-in-Aid for Scientific Research (nos. 17105006, 20750083). We thank the technical laboratory at the Hybrid NanoMaterials Research Center (HyNaM Center) for AFM, SEM, and thermogravimetric-differential thermal analysis measurements and Mr Y Hayasaka for TEM observations.

1 Merhari, L. ed. Hybrid Nanocomposites for Nanotechnology, (Springer, New York, USA, 2009).

2 Mark, J. E., Lee, C. Y- C. \& Biancini, P. A. eds. Hybrid Organic-Inorganic Composites, (American Chemical Society, Washington, D.C., USA, 1994).

3 Dirix, Y., Bastiaansen, C., Caseri, W. \& Smith, P. Oriented pearl-necklace arrays of metallic nanoparticles in polymers: a new route toward polarization-dependent color filters. Adv. Mater. 11, 223 (1999).

4 Haes, A. J., Stuart, D. A., Nie, S. M. \& Van Duyne, R. P. Using solution-phase nanoparticles, surface-confined nanoparticle arrays and single nanoparticles as biological sensing platforms. J. Fluoresc. 14, 355 (2004).
5 Nie, S. \& Emory, S. R. Probing single molecules and single nanoparticles by surfaceenhanced Raman scattering. Science 275, 1102 (1997)

6 Daniel, M. C. \& Astruc, D. Gold nanoparticles: assembly, supramolecular chemistry, quantum-size-related properties, and applications toward biology, catalysis, and nanotechnology. Chem. Rev. 104, 293 (2004).

7 Link, S. \& El-Sayed, M. A. Spectral properties and relaxation dynamics of surface plasmon electronic oscillations in gold and silver nanodots and nanorods. J. Phys. Chem. B 103, 8410 (1999).

8 Heath, J. R., Knobler, C. M. \& Leff, D. V. Pressure/temperature phase diagrams and superlattices of organically functionalized metal nanocrystal monolayers: the influence of particle size, size distribution, and surface passivant. J. Phys. Chem. B 101, 189 (1997).

9 Ohno, K., Koh, K., Tsujii, Y. \& Fukuda, T. Fabrication of ordered arrays of gold nanoparticles coated with high-density polymer brushes. Angew. Chem. Int. Ed. 42, 2751 (2003).

10 Lu, Y., Yin, Y. D., Li, Z. Y. \& Xia, Y. A. Synthesis and self-assembly of Au@SiO 2 coreshell colloids. Nano Lett. 2, 785 (2002)

11 Holzinger, D., Liz-Marzan, L. M. \& Kickelbick, G. Synthesis of core-shell nanoparticles using organic surface-functionalized gold and Au@SiO2 nanoparticles as multifunctional initiators in atom transfer radical polymerization. J. Nanosci. Nanotech. 6, 445 (2006).

12 Enustun, B. V. \& Turkevich, J. Coagulation of colloidal gold. J. Am. Chem. Soc. 85, 3317 (1963).

13 Mine, E., Yamada, A., Kobayashi, Y., Konno, M. \& Liz-Marzan, L. M. Direct coating of gold nanoparticles with silica by a seeded polymerization technique. J. Colloid. Inter. Sci. 264, 385 (2003).

14 Ohno, K., Morinaga, T., Koh, K., Tsujii, Y. \& Fukuda, T. Synthesis of monodispersed silica particles coated with well-defined, high-density polymer brushes by surfaceinitiated atom transfer radical polymerization. Macromolecules 38, 2137 (2005).

15 Fischer, $\mathrm{H}$. The persistent radical effect: a principle for selective radical reactions and living radical polymerizations. Chem. Rev. 101, 3581 (2001).

16 Ejaz, M., Yamamoto, S., Ohno, K., Tsujii, Y. \& Fukuda, T. Controlled graft polymerization of methyl methacrylate on silicon substrate by the combined use of the LangmuirBlodgett and atom transfer radical polymerization techniques. Macromolecules $\mathbf{3 1}$, 5934 (1998).

17 Turkevich, J. \& Kim, G. Palladium: preparation and catalytic properties of particles of uniform size. Science 169, 873 (1970).

18 Turkevich, J., Stevenson, P. C. \& Hiller, J. A study of the nucleation and growth processes in the synthesis of colloidal gold. Discuss. Faraday Soc. 11, 55 (1951).

19 Liu, S. \& Han, M. Synthesis, functionalization, and bioconjugation of monodispersed, silica-coated gold nanoparticles: robust bioprobes. Adv. Funct. Mater. 15, 961 (2005).

20 Ohno, K., Koh, K., Tsujii, Y. \& Fukuda, T. Synthesis of gold nanoparticles coated with well-defined, high-density polymer brushes by surface-initiated living radical polymerization. Macromolecules 35, 8989 (2002).

21 Poupinet, D., Vilanove, R. \& Rondelez, F. Molecular weight dependence of the second virial coefficient for flexible polymer chains in two dimensions. Macromolecules 22, 2491 (1989).

22 Liz-Marzan, L. M., Giersig, M. \& Mulvaney, P. Synthesis of nanosized gold-silica core-shell particles. Langmuir. 12, 4329 (1996).

23 Mitsuishi, M., Matsui, J. \& Miyashita, T. Functional organized molecular assemblies based on polymer nano-sheets. Polym. J. 38, 877 (2006).

24 Matsui, J., Sato, Y., Mikayama, T. \& Miyashita, T. Fabrication of electrochemical transistor based on pi-conjugate polymer Langmuir-Blodgett film. Langmuir. 23, 8602 (2007).

25 Matyjaszewski, K. \& Xia, J. H. Atom transfer radical polymerization. Chem. Rev. 101, 2921 (2001).

26 Parvin, S., Matsui, J., Sato, E. \& Miyashita, T. Side-chain effect on Langmuir and Langmuir-Blodgett film properties of poly( $\mathrm{N}$-alkylmethacrylamide)-coated magnetic nanoparticles. J. Colloid. Inter. Sci. 313, 128 (2007).

27 Parvin, S., Sato, E., Matsui, J. \& Miyashita, T. Surface initiated atom transfer radical polymerization of $\mathrm{N}$-hydroxysuccinimide methacrylate from magnetite surface as precursor for functional polymer shell. Polym. J. 38, 1283 (2006). 